\title{
OBSERVATIONAL STUDIES OF CATACLYSMIC VARIABLE EVOLUTION: OF SAMPLES, BIASES AND SURPRISES
}

\author{
B.T. Gänsicke ${ }^{1}$ \\ RESUMEN
}

Presento un breve informe sobre el estado de tres grandes proyectos observacionales destinados a verificar nuestra actual comprensión de la evolución de variables cataclísmicas (CVs): la selección espectroscópica de las nuevas CVs cn el Hamburg Quasar Survey, la búsqueda de pre-CVs basándose en los colores de Sloan y en la espectroscopía multi-objeto UK Schmidt/6dF y la identificación de CVs que proceden de binarias de rayos X supersuaves, utilizando el "survey" espectroscópico en el ultravioleta lejano del HST/STIS.

\section{ABSTRACT}

I present brief status reports on threc large observational projects that are designed to test our current understanding of the evolution of cataclysmic variables (CVs): The spectroscopic selection of new CVs in the Hamburg Quasar Survey, the search for pre-CVs based on Sloan colours and UK Schmidt/6dF multiobject spectroscopy, and the identification of CVs that descended from supersoft X-ray binaries using a HST/STIS far-ultraviolet spectroscopic survey.

\section{Key Words: NOVAE, CATACLYSMIC VARIABLES - STARS: EVOLUTION}

\section{INTRODUCTION}

The essential idea of the standard model of cataclysmic variable (CV) evolution (disrupted magnetic braking, King 1988) is that CVs evolve to towards shorter periods duc to a combination of angular momentum losses: magnetic braking (dominating in systems with orbital periods $P_{\text {orb }} \gtrsim 3 h$ ) and the less efficient gravitational radiation (dominating in systems with orbital periods $P_{\text {orb }} \lesssim 2 \mathrm{~h}$ ). The standard paradigm of $\mathrm{CV}$ evolution successfully explains the 2-3 $\mathrm{h}$ gap in the observed $\mathrm{CV}$ period distribution. However, most other predictions made by this model are in strong contrast with the properties of the known CV population. Recently, a number of far-reaching modifications for the standard scenario have been proposed (sec Spruit \& Taam 2001, King \& Schenker 2002, Schenker \& King 2002 and Andronov et al. 2003). Unfortunately, none of them has been completely successful in tuning the predictions so that they fully agree with the observations. It is apparent that the disturbing disagreements between theory and observations have a common denominator: the possible impact of selection effects on the currently known population of CVs. In order to quantitatively test any theory of $\mathrm{CV}$ evolution it is necessary to establish a large and unbiased sample of CVs as well as of their progenitors, (pre-CVs, detached white dwarf/late type main sequence stars).

\footnotetext{
${ }^{1}$ Department of Physics, University of Warwick, Coventry CV4 7AL, UK.
}

Such an observational data base will also serve for future improvements of the theory of CV evolution.

\section{CVS FROM THE HQS}

Most CVs have been discovered through one of the following three channels: variability, blue colour, or X-ray emission. We are currently pursuing a large search for CVs based primarily on their spectroscopic properties, using the Hamburg Quasar Survey (HQS; Hagen et al. 1995) as target sample (Gänsicke et al. 2002). The HQS provides an efficient means of discovering CVs that are so far under-represented in the currently known sample of CVs, i.e. weak $\mathrm{X}$-ray emitters, not particularly blue objects, and CVs that show variability with low amplitudes or long recurrence times. A careful study of the CV discovering efficency of the HQS concluded that this survey is especially sensitive to short period systems, such as SU UMa dwarf novae (Gänsicke et al. 2002).

So far, we have discovered 53 new CVs, including a number of fascinating systems (Araujo-Betancor et al. 2004, Gänsicke et al. 2004), and doubling the number of known CVs in the sky area/magnitude range covered by the survey, and measured orbital periods for 29 of these systems (Fig. 1). The simple fact that we do not detect a large number of new short orbital period systems implies that either they do not exist in the quantity predicted by the theories, or that they look very different from the short period CVs we know (e.g. have weak emission lines). Another striking feature of the HQS CV population is 


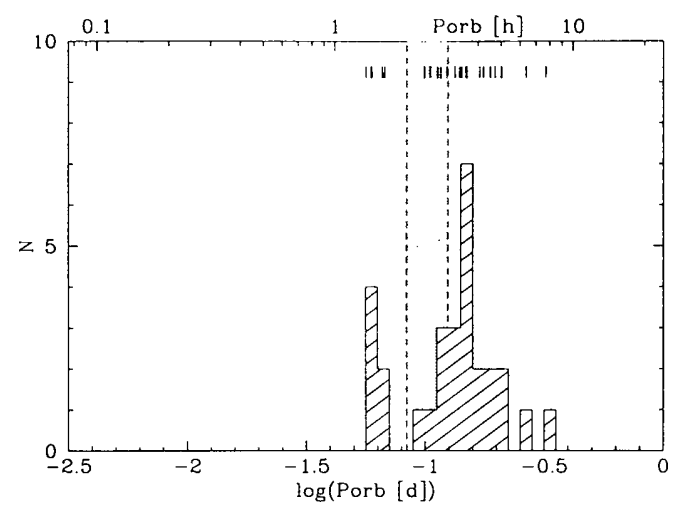

Fig. 1. The period distribution of 29 new CVs from the HQS. An additional $24 \mathrm{HQS}$ CVs are awaiting their period measurement. Shown in gray is the $2-3 \mathrm{~h}$ period gap. Note the concentration of systems in the $3-41$, period range.

the large number systems in the 3-4h orbital period range, most of which (at least 6) are new SW Sex stars (Rodríguez-Gil et al. 2004). Whereas SW Sex stars initially appeared to be the "freaks" among the CVs, they now turn out to be a significant sub-class, and understanding their relation to the global population of CVs is likely to be closely related to a major improvement of our understanding of CV evolution as a whole.

\section{PRE-CVS FROM SLOAN/UKST}

Whereas far more than $1000 \mathrm{CVs}$ are listed by Downes et al. (2001), only a shocking small number of pre-CVs is currently known. Schreiber \& Gänsicke (2003) have analysed in detail the properties of all (30) pre-CVs for which both the orbital period and the white dwarf temperature have been measured, and showed that this population is heavily biased towards binaries containing rather hot (young) white dwarfs $\left(T_{\mathrm{wd}} \gtrsim 15000 \mathrm{~K}\right)$ and low-mass/late-type $\left(M_{\mathrm{sec}} \lesssim 0.4 M_{\odot}\right)$ secondary stars. An important consequence of this bias is that we currently know only a single progenitor for CVs with periods $\gtrsim 4 \mathrm{~h}$ : V471 Tau. Schreiber \& Gänsicke also showed that this bias is a natural result of the way that most pre-CVs were discovered - as a white dwarf in the first place, with some evidence (weak emission lines, eclipses, or ellipsoidal modulation) for a faint companion cropping up later. Among the exceptions is V471 Tau, which was indeed discovered as a spectroscopic binary. Schreiber \& Gänsicke conclude that there ought to be a large population of pre-CVs containing cold (old) and/or early-type secondary stars. We have initiated a large search for pre-CVs with the aim to produce a large and (largely) unbiased sample these systems. We selected our initial target list from Sloan Digital Sky Survey Data Release 1
(Abazajian et al. 2003) by applying the following colour cuts: $u-g<0.8, g-r<1.2$, and redder than the main-sequence. A pilot spectroscopic survey was carried out in October 2003 with the $6 \mathrm{dF}$ on the $1.2 \mathrm{~m}$ UK Schmidt Telescope, covering an area of $\simeq 250 \mathrm{deg}^{2}$ with $g \lesssim 18.5$. In this run we identified 55 new white dwarf/red dwarf binaries, a large fraction of them containing cold white dwarfs (Fig. 2). An independent search for white dwarf/red dwarf binaries was carried out by Raymond et al. (2003), also selecting targets on the base of Sloan colours. but using the Sloan spectrograph for the identification spectroscopy. The different colour selection employed by Raymond et al. leads to a higher fraction of hot white dwarfs in their sample. At the current stage the orbital periods of the systems identified by both projects are yet undetermined, and time-resolved follow-up"spectroscopy/photometry is necessary to distinguish the genuine pre-CVs from wide binaries. Nevertheless, the results obtained so far clearly demonstrate that combining multi-colour target selection with multi-object identification spectroscopy will boost the number of known pre-CVs over the next few years by a large factor.

\section{POST-SUPERSOFT CVS = FAILED SN IA?}

Supersoft X-ray binaries are one likely channel for producing supernovae of type Ia (e.g. Langer et al. 2000). In these systems, contrary to "normal" $\mathrm{CVs}$, the donor star is more massive than the white dwarf, and mass transfer occurs on the thermal time scale of the donor star (Schenker et al. 2002). If the white dwarf fails to grow over the Chandrasekhar limit, the mass ratio will eventually change over $\left(M_{\mathrm{wd}}>M_{\text {donor }}\right)$, and the system will appear as an apparently "normal" CV - with the difference that the donor star is not a main sequence star. but the exposed core of a previously more massive star. Post-supersoft X-ray binaries can, hence, be considered failed SN Ia, and their observational hallmark should be abundances typical of $\mathrm{CNO}$ burning.

Testing the abundances of the donor star in a CV is equivalent to testing the abundances of the material that it is transferring onto the white dwarf, i.e. the abundances of the accretion disc/flow. The natural wavelength range to carry out this test is the far-ultraviolet (FUV), which contains the $\mathrm{NV} \lambda 1240$ and CIV $\lambda 1550$ resonance lines. We are currently carrying out a HST/STIS FUV spectroscopic survey of a large number of CVs, and Gänsicke et al. (2003) presented the strong evidence that EY Cyg, BZ UN la, EI Pse (1RXS J232953.9+1)62814) and V396 Hya (CE 315) are post-supersoft X-ray binaries. Since the publication of these results, our 

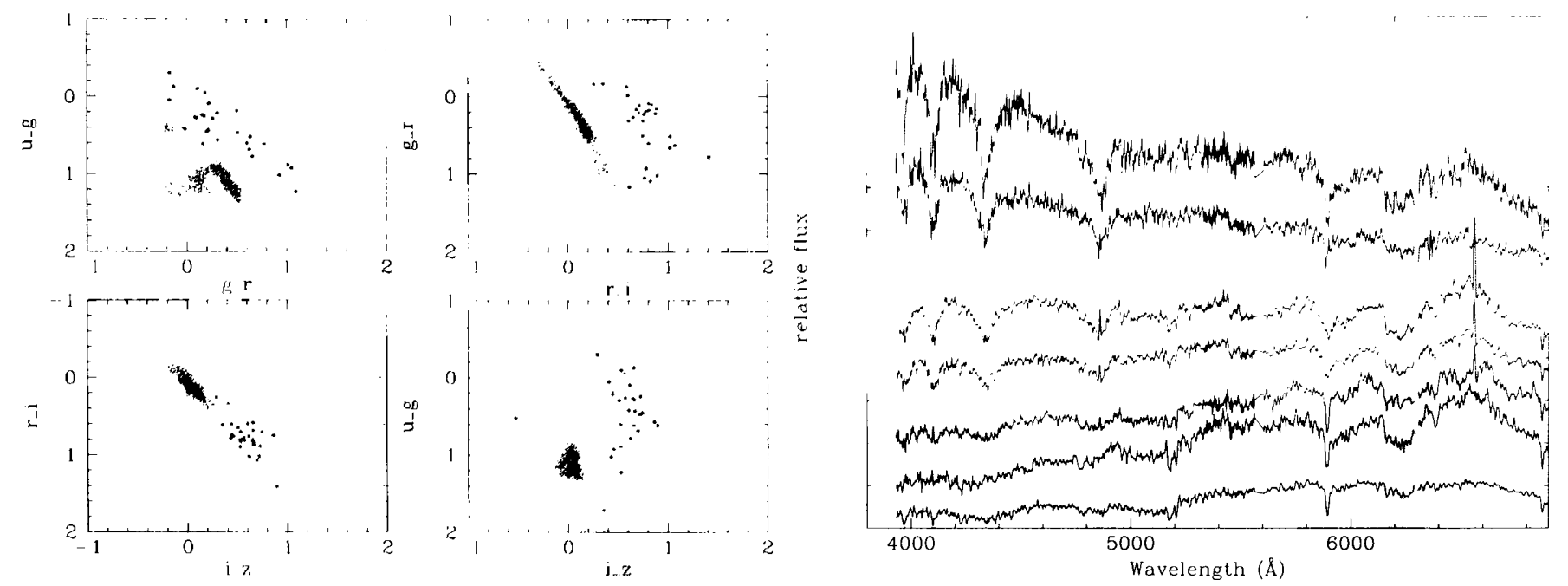

Fig. 2. Left panel: Colour-colour diaglanus of the white dwarf/red dwarf binaries identified from UKST/6dF observations. 'The small gray points mprent the location of the main sequence and white dwarf population. Right panel: Sample spectra of the UKS'T/6all' whitc dwarf/red dwarf binaries. The wavelength of the Balmer lines and the most prominent $\mathrm{TiO}$ absorption are shown in gray. The spectia ale sorted by decreasing white dwarf contribution.

$H S T$ /STIS survey lias muveiled three adelitional systems witl significantly culranced N/C cmission line flux ratios: QZSer, (SS Pav, and ('W1045+525. The fraction of liknly failed SN Ia in lhe HST/STIS surrey is $\sim 12 \%$ which is within the range predicted by the evolutionary models. However, considering the still rather small total number of systems observed in this survey, the stat istical significance of this fraction is too low for a definite conclusion. Apart from the systems mentioned hore. AE Aqr, V13090 Ori, TX ('ol. BY ('am, MN Hya, and GP Com have been shown to exhibit strong $\mathrm{N} /$ (' $^{\prime}$ enhancement. The 14 post-supersoft, ( $V$ s known so far span a large range in orbital periods (47-661 min) and CV subclasses (clwat novale nova-like variables. polars, intermediate polars. $A M\left({ }^{\prime} \mathrm{V}\right.$ systems). This underlines the fact that the olserverl abundance anomalies are likely to bo related to a gencral crolutionary effece rather than to the specific properties of a small subgroup of $\mathrm{CVs}$.

I am grateful to all my fricuds and colleagues who have contributed to this endeavour ower the last years: Sofia Araujo-Betancor, Heinz Barwig, Klaus Beuermann. Domitilla de Martino. Dieter Engels, Bob Fried. Hans Hagen, Emilios Harlaftis, Ivan Hubeny. Christian Knigge, Knox Long, Tom Marsh. Ronald Mennickent, Daisaku Nogami, Pablo Rodríguez-Gil, Linda Schmidtobreick, Matthias Schreiber. Robert Schwarz. Andreas Staude, Ed Sion. Paula Szkody: Claus Tappert, John Thorstensen.

\section{REFERENCES}

Abazajian, K., Adelman-McCarthy, J. K., Agüeros, M. A., et al. 2003, 126, 2081

Andronov, N., Pinsonneault, M. \& Sills, A. 2003, ApJ, 582,358

Araujo-Betancor, S., Gänsicke, B. T., Hagen, H.-J., et al. 2004 , this volume

Downes, R. A., Webbink, R. F., Shara, M. M., et al. 2001, PASP, 113, 764

Gänsicke, B. T., Hagen, H.-J. \& Engels, D. 2002, ASP Conf. Ser. 261, 190

Gänsicke, B. T., Szkody, P., de Martino, D., et al. 2003 , ApJ, 594, 443

Gänsicke, B. T.. Araujo-Betancor, S., Hagen, H.-J., et al. 2004 , this volume

Hagen, H.-J., Groote, D., Engels, D. \& Reimers, D. 1995, Ad AS, 111,195

King, A. R. 1988, QJRAS, 29,1

King, A. R. \& Schenker, K. 2002, ASP Conf. Ser. 261, 233

Langer, N., Deutschmann, A., Wellstein, S., \& Höflich, P. $2000, A \& A, 362,1046$

Raymond, S. N., Szkody, P., Hawley, S. L., et al. 2003, AJ. 125,2621

Rodríguez-Gil, P., Gänsicke, B. T., Hagen, H.-J., et al. 2004. Ad A submitted

Schenker, K. \& King, A. R. 2002, ASP Conf. Ser. 261, $242-251$

Schenker, K., King, A. R., Kolb, U., et al. 2002, MNRAS, 337.1105

Schreiber, M. R. \& Gänsicke, B. T. 2003, A\&A, 406, 305

Spruit, H. C.\& Taam, R. E. 2001. ApJ, 548, 900 